12

\title{
Эффект обратной релаксации и структура пористых электродов суперконденсаторов
}

\author{
(C) М.Е. Компан, В.Г. Малышкин
}

Физико-технический институт им. А.Ф. Иофрфе РАН, Санкт-Петербург, Россия

E-mail: kompan@mail.ioffe.ru

Поступило в Редакцию 15 мая 2018 г.

Исследовано самовосстановление напряжения на суперконденсаторах различных марок. Показана связь параметров этого процесса с характеристиками пористой структуры электродов суперконденсаторов. Предложенный подход позволяет экспериментально определить долю емкости суперконденсатора, активную в импульсном процессе заданной длительности.

DOI: 10.21883/PJTF.2019.02.47224.17384

Электроды энергонакопительных устройств (суперконденсаторов, Li-ионных аккумуляторов и др.), как правило, создаются из пористых материалов. У суперконденсаторов накопление заряда и энергии локализовано на границе раздела электрод-электролит. Поэтому для материалов электродов используются материалы с развитой системой пор: как правило, различные типы пористых углей, обладающих за счет присутствия пор нанометрового размера развитой поверхностью порядка $1500-2000 \mathrm{~m}^{2} / \mathrm{g}$ [1]. Структура пористого материала определяет мощностные параметры суперконденсаторов и, как показано далее, в том числе обусловливает появление эффекта обратной релаксации, которому посвящена настоящая работа.

В большинстве пористых углеродных материалов, используемых для электродов, нанопористая структура либо формируется спонтанно, без прямого вмешательства технологов (например, в углях типа CDC [2]), либо наследуется от исходных растительных материалов (угли типа NORIT и аналогичные [3]). Известны также попытки получения углеродных материалов с контролируемой иерархической структурой. В некоторых из упомянутых работ пористый углеродный материал получали, применяя дополнительный материал с заданной системой пор в качестве шаблона, „жертвенный“ материал впоследствии удалялся. Другой подход к получению желательной структуры пор основан на сегрегации фаз [4].

Диагностика пористой структуры уже полученного углеродного материала весьма несовершенна. Измерения удельной поверхности пор по методу Брунауэра-Эммета-Теллера [5] применяются для последующего расчета распределения пор по размерам. Однако используемый метод расчета (теория функционала плотности) требует априорных представлений о форме пор и совсем не дает информации о распределении пор по доступности (по времени заряда/разряда).

Наиболее развитый электрический метод исследования пористых электродов суперконденсаторов - импедансная спектроскопия - требует предварительных предположений об эквивалентной электрической схеме объекта [6], поскольку одному и тому же набору экспериментальных данных могут соответствовать различные эквивалентные схемы. Кроме того, для интерпретации данных в импедансометрии используются формальные типы элементов (Варбурга, Геришера и т.д.), которые зачастую могут не прояснять физической картины происходящего в объекте [6]. Также важно, что импеданс суперконденсаторов нелинеен, зависит от накопленного в них заряда, что в случае глубокого разряда усложняет расчеты и делает их результаты плохо соотносимыми с реальными параметрами [7].

В настоящей работе сделана попытка разработки простого и эффективного метода оценки параметров суперконденсаторов, характеризующих их быстродействие. Важно, что измерения проводятся в типичном для приложений сильнотоковом режиме. В основу метода положен эффект обратной релаксации, проявляющийся при быстром разряде суперконденсаторов. Эффект заключается в медленном росте напряжения на выходных клеммах после быстрого (частичного или полного) разряда. Эффект обусловлен наличием в структуре суперконденсатора распределенных $R C$-цепей. В работе [8] упоминается о наблюдении такого эффекта, однако он не исследовался и не рассматривался как источник информации о пористой структуре.

В соответствии с развитым подходом будем подразделять систему пор в электродах на быструю (доступную) подсистему и медленную подсистему, которая не участвует в быстрых процессах заряд/разряд (см. вставку на рис. 1). Тогда при кратковременном подключении нагрузки к входным клеммам суперконденсатора разрядится только конденсатор $C_{1}$, а после размыкания цепи начнется процесс выравнивания напряжений на емкостях $C_{1}$ и $C_{2}$ через сопротивление $R_{2}$. В рамках модели процессы при обратной релаксации представляют собой перераспределение зарядов между быстрой и медленной подсистемами $\left(Q_{C 1}\right.$ и $Q_{C 2}$ соответственно). Поскольку $\Delta Q_{C 1}=\Delta Q_{C 2}$, отношение емкостей быстрой и медленной подсистем пор просто находится из эксперимен- 


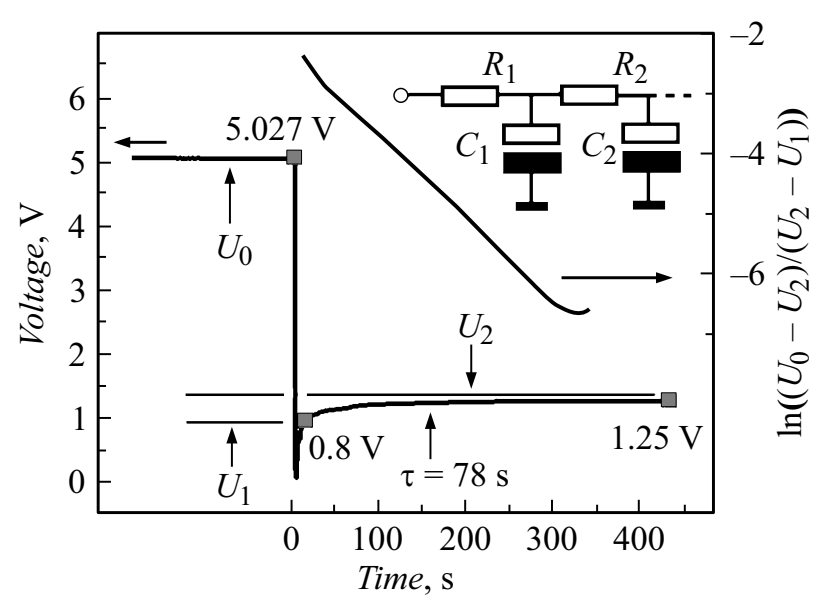

Pис. 1. Зависимость напряжения на многосекционном суперконденсаторе К58-12 0.1 F с твердым кристаллическим электролитом после кратковременного включения нагрузки (по левой оси). Показаны напряжения $U_{0}, U_{1}$ и $U_{2}$, использующиеся в расчетах. Квадратами обозначены точки на экспериментальной зависимости, в которых брались численные значения для расчетов; рядом указана измеренная величина. По правой оси - зависимость логарифма величины $\left(U_{0}-U_{2}\right) /\left(U_{2}-U_{1}\right)$ от времени. Момент времени $t=0$ совпадает с моментом включения нагрузки. На вставке - двухзвенная $R C$-цепь, соответствующая разделению емкости суперконденсатора на „быструю“ и „медленную“ компоненты.

тально измеряемых величин $U_{0}, U_{2}, U_{1}$ (обозначены на рис. 1)

$$
C_{1}=C_{2}\left(\left(U_{0}-U_{2}\right) /\left(U_{2}-U_{1}\right)\right) .
$$

Номинальная емкость суперконденсатора соответствует сумме $\left(C_{1}+C_{2}\right)$. Измерение характеристик процесса обратной релаксации интересно тем, что из данных при таких измерениях исключается величина входного сопротивления $R_{1}$, а ток через сопротивление $R_{2}$ течет в сторону, противоположную той, в которую он течет при зарядке суперконденсатора. Этим предложенный процесс измерения принципиально отличается от протекающего при обычной подаче тока через входные клеммы, что позволяет получать независимую информацию о внутренних процессах релаксации. Наиболее важной величиной при этом является емкость $C_{1}$, доступная в режиме получения максимума мощности. Тем самым модель позволяет анализировать эффективность использования пористой среды электродов.

Продемонстрируем методику обратной релаксации в экспериментах с серийными суперконденсаторами нескольких типов (рис. 1, 2). Эксперименты проводились с суперконденсаторами следующих типов: AVX 1.0 F, 2.7 V; AVX 3.3 F, 2.7 V; Aeroce A 1.0 F, 2.7 V; K58-12 0.1 F, $7.5 \mathrm{~V}$. Последний из указанных типов - многосекционный суперконденсатор с твердым кристаллическим электролитом $\mathrm{RbAg}_{4} \mathrm{I}_{5}$.
На рис. 1 по левой оси отложено изменение напряжения на суперконденсаторе К58-12 0.1 F со временем в процессе обратной релаксации. Начало отчета времени по горизонтальной оси совпадает с включением нагрузки. Точки на кривой, где измерялись величины напряжений, обозначены квадратами. Видно, что за время включения нагрузки напряжение на конденсаторе падает почти до нуля; затем, с выключением нагрузки, напряжение быстро частично восстанавливается, так как суперконденсатор не был полностью разряжен. Затем начинается медленный рост напряжения до величины, обозначенной на рис. 1 как $U_{2}$. Рассчитанная по результатам измерений доступная „быстрая“ компонента этого конденсатора составляет $0.08 \mathrm{~F}$.

На этом же рисунке (правая ось) приведена зависимость логарифма релаксирующей величины $\ln \left(U_{2}-U(t)\right)$ от времени $(t)$. Видно, что график логарифма здесь представляет собой прямую, что указывает на одно конкретное время релаксационного процесса (в данном случае $78 \mathrm{~s}$ ). Горизонтальная ось времени на этом рисунке единая для двух кривых.

Аналогичное поведение демонстрирует и суперконденсатор AVX 3.3 F с жидким электролитом (рис. 2). На вставке к этому рисунку показана зависимость напряжения от времени в растянутом временно́м масштабе, так что просматривается временно́й интервал нагрузки. Метки, где измерялись величины напряжений, такие же, как на рис. 1. Правая ось на рис. 2 соответствует зависимости от времени логарифма отличия напряжения от асимптотического $\ln \left(U_{2}-U(t)\right)$. Для данного суперконденсатора наклон этой кривой меняется со временем, что свидетельствует о наложении нескольких релаксационных процессов. Величина „быстрой“ компоненты емкости составила $2.8 \mathrm{~F}$.

Можно показать, что величина и сопротивление нагрузки (при некоторых ограничениях) слабо влияют на результат измерений, что делает предложенный метод

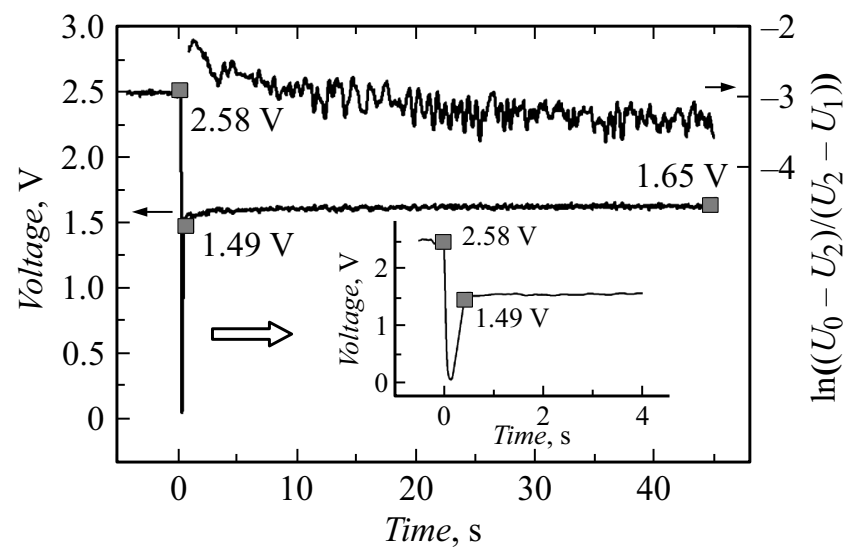

Рис. 2. То же, что на рис. 1 , для суперконденсатора AVX $3.3 \mathrm{~F}$ с жидким электролитом. На вставке - фрагмент основной зависимости в растянутом временно́м масштабе, показывающий зависимость напряжения на суперконденсаторе во время включения нагрузки. 
Результаты расчетов величин напряжений $U_{2}, U_{1}$ и величины $\left(U_{0}-U_{2}\right) /\left(U_{2}-U_{1}\right)$ на модельном суперконденсаторе

\begin{tabular}{l|c|c|c|c}
\hline$\tau / R_{2} C_{2}$ & $U_{0}, \mathrm{~V}$ & $U_{1}, \mathrm{~V}$ & $U_{2}, \mathrm{~V}$ & $\left(U_{0}-U_{2}\right) /\left(U_{2}-U_{1}\right)$ \\
\hline 0.01 & 1 & 0.980203 & 0.981672 & 12.4720 \\
0.025 & 1 & 0.951255 & 0.954859 & 12.5224 \\
0.05 & 1 & 0.9049320 & 0.911917 & 12.6085 \\
0.1 & 1 & 0.8190777 & 0.832201 & 12.7864 \\
0.25 & 1 & 0.6082554 & 0.635524 & 13.3658 \\
0.5 & 1 & 0.3726584 & 0.413140 & 14.4969
\end{tabular}

характеризации пористого материала удобным и корректным для практического использования. В таблице представлены результаты модельных расчетов величины отношения „быстрой“ и „медленной“ емкостей, получаемые при измерениях с разными временами включения нагрузки. Из данных таблицы видна очень слабая зависимость отношения $C_{1} / C_{2}$ (последний столбец) от времени включения нагрузки (первый столбец). Начальное напряжение суперконденсатора в расчетах принималось равным $1 \mathrm{~V}$.

Представляет интерес кинетика обратной релаксации. Если в режиме обратной релаксации наблюдается одно время (как на рис. 1), то это, скорее всего, означает наличие массива пор одинаковой структуры, вероятно унаследованных от растительного сырья. Наличие распределения времен обратной релаксации (как на рис. 2), скорее, свидетельствует о случайном процессе порообразования.

Таким образом, исследован процесс обратной релаксации напряжения на нескольких типах суперконденсаторов, предложена концепция быстрой и медленной подсистем накопления заряда, которая позволяет по экспериментальным характеристикам оценить долю „быстрой“6 емкости таких устройств.

\section{Список литературы}

[1] Ji H., Zhao X., Qiao Z., Jung J., Zhu Y., Lu Y., Zhang L.L., MacDonald A.H., Ruoff R.S. // Nature Commun. 2014. V. 5. P. 3317.

[2] Oschatz M., Boukhalf S., Nickel W., Hofmann J.P., Fischer C., Yushin G., Kaskel S. // Carbon. 2017. V. 113. P. 283-291.

[3] Abioye A.M., Ani F.N. // Renewable Sustainable Energy Rev. 2015. V. 52. P. $1282-1293$.

[4] Borenstein A., Hanna O., Attias R., Luski S., Brousse T., Aurbach D. // J. Mater. Chem. A. 2017. V. 5. P. 12653-12672.

[5] Brunauer S., Emmelt P.H., Teller E. // J. Am. Chem. Soc. 1938. V. 60. P. 309-319.

[6] Impedance spectroscopy: theory, experiment, and applications / Eds E. Barsoukov, J.R. Macdonald. 2nd ed. Wiley-Interscience, 2005. $616 \mathrm{p}$.

[7] Компан М.Е., Кузнецов В.П., Малышкин В.Г. // ЖТФ. 2010. T. 80. B. 5. C. 100-106.

[8] Fletcher S., Kirkpatrick I., Dring R., Puttock R., Thring R., Howroyd S. // J. Power Sources. 2017. V. 345. P. 247-253. 